\title{
Comparison between posterior dynamic stabilization and posterior lumbar interbody fusion in the treatment of degenerative disc disease: a prospective cohort study
}

Haodong Fei ${ }^{1+}$, Jiang $\mathrm{Xu}^{2+}$, Shouguo Wang ${ }^{1}$, Yue Xie ${ }^{1}$, Feng $\mathrm{Ji}^{1^{*}}$ and Yongyi $\mathrm{Xu}^{1}$

\begin{abstract}
Background: Few studies compared radiographic and clinical outcomes between posterior dynamic stabilization (PDS) and posterior lumbar intervertebral fusion (PLIF) in treating degenerative disc disease (DDD).

Methods: A total of 176 consecutive patients who underwent posterior instrumented spinal surgery for degenerative disc disease between January 2007 and January 2009 were prospectively divided into two groups_-PDS and PLIF. All patients included in the analysis were followed up for 3 years. Demographic distribution, perioperative complications, and radiographic and clinical outcomes were compared between the two groups.

Results: The amount of intraoperative blood loss and drained volume was significantly greater in the PLIF group compared with the PDS group $(881.1 \mathrm{ml}$ versus $737.4 \mathrm{ml}, p=0.004)$. The length of stay of patients who had PLIF surgery (20.9 days) was significantly longer $(p=0.033$ ) than that of patients who underwent PDS surgery (18.9 days). Patients with PLIF surgery had higher total costs than those with PDS surgery (US\$12826.8 versus US\$11654.5, $p=0.002$ ). No statistically significant differences existed in back visual analogue scale (VAS), leg VAS, or Oswestry disability index (ODI) scores between the PDS and PLIF groups of patients at each time point.

Conclusions: Compared with PLIF, PDS have advantages on blood loss, length of stay in hospital, total charges, and radiographic outcomes, but no advantages on leg and back VAS or ODI scores. High-quality randomized controlled trials are still required in the future.
\end{abstract}

Keywords: Dynamic stabilization, Dynesys, Lumbar spine, Posterior lumbar interbody fusion, Degenerative disc disease

\section{Introduction}

Instrumented fusion in the treatment of degenerative conditions of the lumbar spine is known to have potential complications such as pseudoarthrosis, nonunion, instrumentation failure, infection, donor site pain, and adjacent segment disease [1-3]. The Dynesys Spinal Stabilization System (Zimmer, Inc., Minneapolis, MN, USA) uses pedicle screws, polyethylene-terephthalate cords, and polycarbonate urethane spacers to stabilize a functional spinal unit. The concept of these devices is to reduce the load

\footnotetext{
*Correspondence: habest126@126.com

${ }^{\dagger}$ Equal contributors

'Department of Orthopaedics, Huai'an First People's Hospital, Nanjing Medical University, Huai'an, Jiangsu 223300, People's Republic of China Full list of author information is available at the end of the article
}

and restrict the motion of the affected level to stop or delay its degeneration. While studies have indicated positive outcomes with improved Oswestry disability index (ODI) scores and visual analogue scale (VAS) pain scores, as well as shorter recovery time than the fusion for patients with degenerative disc disease (DDD) of the lumbar spine treated with the Dynesys system [4-6], to our knowledge, literature is lacking in studying differences in radiographic and clinical outcomes between posterior dynamic stabilization (PDS) and posterior lumbar interbody fusion (PLIF).

This prospective cohort study was designed to evaluate radiographic and clinical outcomes between posterior dynamic stabilization and posterior lumbar interbody fusion in patients with degenerative disc disease. Furthermore, 
we hypothesized that PDS would lead to shortened length of hospital stay, reduced medical costs, and fewer perioperative complications.

\section{Materials and methods}

The study protocol was approved by the local institutional review board and ethics committee. Informed consent was obtained from all study participants. A total of 176 consecutive patients undergoing posterior instrumented spinal surgery for degenerative disc disease between January 2007 and January 2009 at a hospital were prospectively divided into two groups according to their clinic sequences. There were 51 males and 44 females subject to PDS, with the mean age of 47.3 years. The implanted level included L2-L5 in 1 case, L3-L4 in 5 cases, L3-L5 in 7 cases, L4-L5 in 45 cases, L4-S1 in 10 cases, and L5-S1 in 27 cases. In the PLIF group, there were 40 males and 41 females, with the mean age of 52.9 years. The implanted level included L2-L5 in 2 cases, L3-L4 in 4 cases, L3-L5 in 5 cases, L4-L5 in 47 cases, L4-S1 in 13 cases, and L5-S1 in 10 cases. The statistical information of patients, including comorbidities, was presented in Table 1. Degenerative lumbar disc disease had been diagnosed in all of these patients, and they all suffered from severe neurogenic claudication and leg, buttock, or groin pain with back pain that was aggravated in sitting or lumbar flexion and relieved by upright position. In all cases, conservative treatment had failed to control these patients' symptoms. Patients who

Table 1 Demographics of the groups

\begin{tabular}{llll}
\hline Characteristics & $\begin{array}{l}\text { Dynesys group } \\
(n=95)\end{array}$ & $\begin{array}{l}\text { PLIF group } \\
(n=81)\end{array}$ & $p$ \\
\hline Age (years) ${ }^{\mathrm{a}}$ & $47.3 \pm 12.9$ & $52.9 \pm 11.2$ & 0.002 \\
Sex & 51 & 40 & 0.569 \\
Male & 44 & 41 & \\
Female & $26.9 \pm 3.9$ & $22.9 \pm 3.3$ & $<0.001$ \\
BMl (kg/m²) & & & 0.136 \\
Comorbid medical conditions & & 17 & \\
Yes & 12 & 64 & \\
No & 83 & & 0.158 \\
Implanted level, $n$ (\%) & & $2(2.5)$ & \\
L2-L5 & $1(1.1)$ & $4(4.9)$ & \\
L3-L4 & $5(5.3)$ & $5(6.2)$ & \\
L3-L5 & $7(7.4)$ & $47(58.0)$ & \\
L4-L5 & $45(47.4)$ & $13(16.0)$ & \\
L4-S1 & $10(10.5)$ & $10(12.3)$ & \\
L5-S1 & $27(28.4)$ & &
\end{tabular}

Comorbid medical conditions include cardiovascular disorders, respiratory diseases, digestive system disorders, diabetes mellitus, renal insufficiency $B M I$ body mass index

${ }^{\mathrm{a}}$ Data are displayed as mean $\pm \mathrm{SD}$ underwent surgical treatment for nondegenerative conditions (trauma, tumor, or infection) were excluded. We also excluded patients with osteoporosis (bone mineral density T-score less than -2.5) and those who had undergone previous lumbar fusion surgery and those with degenerative deformity.

On admission to the hospital for the surgery, all patients underwent re-examination including routine lumbar posterior-anterior and flexion-extension position radiographs, lumbar computed tomography (CT), and magnetic resonance imaging (MRI); lumbar myelography was provided for some patients to ascertain whether there was complicated lumbar intervertebral disc protrusion, canal stenosis, or spondylolisthesis. A summary of findings and the clinical history were then documented on a standardized evaluation form and entered into the hospital's computerized patient records system.

Dynesys implantation was performed in the prone position. A midline approach, with dissection of the erector spinae muscles, provided access to the bony anatomy of the lumbar spine. If indicated, decompression of the spinal canal was performed first. Insertion of the pedicle screws was carried out under radiographic control using a $\mathrm{C}$-arm. The polycarbonate urethane spacer was cut according to the measured distance between the screws, with the length being chosen to compensate for any existing lordosis or kyphosis. The central cord and the spacer were then locked within the screw heads. A soft brace was administered after surgery until wound healing had occurred.

PLIF was performed in a standard manner. Where required, extensive decompression and facetectomy were performed for cage insertion. Autologous bone graft using decompressed bone chips was used within and around the cage, followed by pedicle screw instrumentation and fixation. A posterolateral fusion was also performed for these patients. Surgery was performed by a single senior surgeon. Blood loss was estimated through the evaluation of the amount of blood in the suction canister and that in soaked lap pads. The surgery was performed with the patient under hypotensive anesthesia in which systolic blood pressure was kept lower than $90 \mathrm{~mm} \mathrm{Hg}$. All wounds were closed over hemovac drains which were placed on continuous suction. Drains were discontinued no sooner than postoperative day 3 , with the output less than $100 \mathrm{ml}$ over $24 \mathrm{~h}$. The same blood transfusion guidelines were used for all patients. Allogenic blood transfusion was performed if hemoglobin decreased to less than $7.0 \mathrm{mg} / \mathrm{dL}$ or if anemic symptoms developed, such as a decrease in blood pressure to less than $100 \mathrm{mmHg}$ systolic, tachycardia greater than 100 beats/min, or a low urine output less than $30 \mathrm{~mL} / \mathrm{h}$, even after initial fluid challenge with $500 \mathrm{~mL}$ normal saline in patients with a hemoglobin level between 7.0 and $8.0 \mathrm{mg} / \mathrm{dL}[7,8]$. 
Information was compiled about perioperative complications occurring within 30 days after operation, including surgical complications (postoperative bleeding, wound breakdown, infection, implant failure, neurologic compromise, etc.) and nonsurgical complications (pulmonary disease, arrhythmia, hemodynamic instability requiring inotropic support, etc.). All patients had at least 3 years of follow-up (3.0-5.5 years) physical and radiographic examinations postoperatively.

Data collected included flexion, extension, neutral radiographs, range of motion (ROM) in sagittal view, anterior and posterior disc heights, ODI score, VAS pain score, preoperative BMD, and total charges. Total charge was defined as hospital charges during index hospitalization alone, not including costs for follow-up care and readmission. Radiographs were measured and analyzed by two experienced spine surgeons. Flexion and extension views were taken with patients in the lateral position. The ROM in the sagittal (flexion-extension) view was obtained by the following formula: ROM sagittal = angle (extension) angle (flexion). Disc height was determined on radiographs taken with the patient in the neutral position and was assessed by measurement of lines drawn at the most prominent points of the endplate anteriorly and posteriorly. Total disc height measured from L1 to S1 was the sum of the disc height at each level. VAS scores were determined on a scale ranging from 0 (no pain) to 100 (worst pain imaginable).

In the PLIF group, fusion status was recorded for each surgically treated segment at each follow-up interval using $\mathrm{X}$-ray film. The level was regarded as fused if there was radiographic evidence of bone bridging the disc space with no lucency. For patients undergoing multiple-level fusion, all surgically treated segments needed to be fused for the patient to be considered as a fusion success.

The data analysis was conducted using SPSS version 19.0 (Chicago, IL, USA). Normal distribution was validated by Shapiro-Wilk test. Continuous data were compared between the PDS and PLIF groups through Student's $t$ test, whereas discontinuous data were analyzed through the chi-squared test. Fisher's exact test was conducted for small data subsets $(n<5)$. All significance tests were twotailed, with $p<0.05$ representing statistical significance. Post hoc power analyses were conducted for the change in VAS, ODI scores, ROM, and anterior disc height and posterior disc height in the PDS and PLIF groups.

\section{Results}

\section{Surgical characteristics}

All variables were in normal distribution. There were no significant differences in mean operation time, mean perioperative red blood cell transfusion unit, and perioperative complications between the two groups (Table 2). However, the mean amount of intraoperative blood loss and drained
Table 2 Comparing variables in patients with posterior dynamic stabilization (PDS) and with posterior lumbar interbody fusion (PLIF)

\begin{tabular}{llll}
\hline Characteristics & Dynesys group $(n=95)$ & PLIF group $(n=81)$ & $p$ \\
\hline Op time $(\mathrm{min})^{\mathrm{a}}$ & $162.3 \pm 41.4$ & $167.3 \pm 37.2$ & 0.404 \\
EBL $(\mathrm{mL})^{\mathrm{a}}$ & $737.4 \pm 307.2$ & $881.1 \pm 373.9$ & 0.004 \\
${\text { Transfusion }(\mathrm{U} / \mathrm{pt})^{\mathrm{a}}}^{\mathrm{a}}$ & $0.12 \pm 0.79$ & $0.09 \pm 0.51$ & 0.869 \\
${\text { Length of stay }(\mathrm{d})^{\mathrm{a}}}^{\mathrm{a}}$ & $18.9 \pm 5.3$ & $20.9 \pm 6.9$ & 0.033 \\
${\text { Total charge }(\mathrm{USD})^{\mathrm{a}}}$ & $11654.5 \pm 1889.3$ & $12826.8 \pm 2946.8$ & 0.002 \\
Complication, $n(\%)$ & $2(2.1)$ & $4(4.9)$ & 0.416 \\
\hline
\end{tabular}

Op time operation time, EBL estimated blood loss, $\mathrm{U}$ blood units: $U / p t$ units per patient

${ }^{\mathrm{a}}$ Data are displayed as mean $\pm \mathrm{SD}$

volume was significantly greater in the PLIF group compared with the PDS group (881.1 $\mathrm{ml}$ versus $737.4 \mathrm{ml}, p=$ 0.004); in addition, patients who had PLIF surgery had a significantly longer mean length of stay (20.9 days) than patients who underwent PDS surgery (18.9 days) ( $p=0.033)$. Mean total charges of patients with PLIF surgery were higher than that of patients with PDS surgery (US\$12,826.8 versus US\$11,654.5, $p=0.002$ ).

\section{Clinical outcomes}

In the PDS group, the mean VAS scores for back pain obtained preoperatively 1 year after surgery and in the final follow-up visit were $43.7 \pm 14.5,19.5 \pm 11.7$, and $10.6 \pm 9.1$, respectively. The corresponding mean VAS scores for leg pain were $51.6 \pm 15.7,13.2 \pm 8.9$, and $6.7 \pm$ 8.3. The corresponding ODI scores were $57.1 \pm 7.7,33.5 \pm$ 6.6, and 25.9 \pm 5.7 . In the PLIF group, the mean VAS scores for back pain obtained preoperatively 1 year after surgery and in the final follow-up visit were $45.3 \pm 13.9$, $18.6 \pm 11.3$, and $11.4 \pm 8.5$, respectively. The corresponding mean VAS scores for leg pain were $52.3 \pm 16.3,11.5 \pm$ 8.2 , and 7.1 \pm 7.5 . The corresponding ODI scores were $56.1 \pm 8.1,33.9 \pm 7.1$, and $24.9 \pm 5.9$. In both groups, VAS and ODI scores decreased after surgery, and there was a statistically significant difference between preoperative and final follow-up scores for back VAS, leg VAS, and ODI scores $(p<0.001)$. However, no statistically significant differences can be seen in back VAS, leg VAS, or ODI scores between the PDS and PLIF groups of patients at each time point (Table 3 ).

\section{Radiological outcomes Range of motion}

ROM values of the implanted segment and L1-S1 levels were measured preoperatively, 1 year after surgery and in the final follow-up visit. There were no significant differences in the mean ROM values for the implanted segments and the mean ROM values for the L1-S1 levels between patients in the PDS group and PLIF group preoperatively $\left(6.8^{\circ} \pm 2.7^{\circ}\right.$ versus $6.6^{\circ} \pm 3.0^{\circ}, p=0.579 ; 18.1^{\circ} \pm$ 
Table 3 Clinical outcome

\begin{tabular}{llll}
\hline & Dynesys group $(n=95)$ & PLIF group $(n=81)$ & $p$ \\
\hline ODI (\%) & & & \\
Pre op & $57.1 \pm 7.7$ & $56.1 \pm 8.1$ & 0.391 \\
Mid-term & $33.5 \pm 6.6$ & $33.9 \pm 7.1$ & 0.738 \\
Final & $25.9 \pm 5.7$ & $24.9 \pm 5.9$ & 0.271 \\
VAS $_{\text {back }}(\mathrm{mm})^{\mathrm{a}}$ & & & \\
Pre op & $43.7 \pm 14.5$ & $45.3 \pm 13.9$ & 0.451 \\
Mid-term & $19.5 \pm 11.7$ & $18.6 \pm 11.3$ & 0.633 \\
Final & $10.6 \pm 9.1$ & $11.4 \pm 8.5$ & 0.568 \\
VAS leg $(\mathrm{mm})^{\mathrm{a}}$ & & & \\
Pre op & $51.6 \pm 15.7$ & $52.3 \pm 16.3$ & 0.752 \\
Mid-term & $13.2 \pm 8.9$ & $11.5 \pm 8.2$ & 0.199 \\
Final & $6.7 \pm 8.3$ & $7.1 \pm 7.5$ & 0.782 \\
\hline VAS visulana & & &
\end{tabular}

VAS visual analogue scale, ODI Oswestry disability index, VASback VAS score for back pain, VASleg VAS score for leg

${ }^{a}$ Data are displayed as mean \pm SD

$5.9^{\circ}$ versus $17.9^{\circ} \pm 6.4^{\circ}, p=0.852$ ) (Table 4 ). However, there were statistical differences in the mean ROM values of the implanted segment and L1-S1 levels between the PDS group and the control group $\left(3.5^{\circ} \pm 1.2^{\circ}\right.$ versus $0.9^{\circ} \pm 0.9^{\circ}$, $p<0.001 ; 15.3^{\circ} \pm 4.9^{\circ}$ versus $13.1^{\circ} \pm 3.3^{\circ}, p=0.001$ ) (Table 5) 1 year after surgery. In the final follow-up visit, the mean ROM values of the implanted segment and L1-S1 levels in the PDS group were significantly higher than that in the control group $\left(3.7^{\circ} \pm 1.4^{\circ}\right.$ versus $0.6^{\circ} \pm 0.6^{\circ}, p<0.001$; $15.8^{\circ} \pm 4.7^{\circ}$ versus $13.6^{\circ} \pm 3.4^{\circ}, p<0.001$ ) (Table 6).

\section{Posterior disc height}

Posterior disc height was assessed with standing neutral lateral radiographs preoperatively, 1 year after surgery and in the final follow-up visit for both groups. The mean

Table 4 Preoperative radiographic outcome

\begin{tabular}{|c|c|c|c|}
\hline & $\begin{array}{l}\text { Dynesys group } \\
(n=95)\end{array}$ & $\begin{array}{l}\text { PLIF group } \\
(n=81)\end{array}$ & $p$ \\
\hline \multicolumn{4}{|l|}{$\mathrm{ROM}\left({ }^{\circ}\right)^{\mathrm{a}}$} \\
\hline Operated level & $6.8 \pm 2.7$ & $6.6 \pm 3.0$ & 0.579 \\
\hline L1-S1 & $18.1 \pm 5.9$ & $17.9 \pm 6.4$ & 0.852 \\
\hline \multicolumn{4}{|c|}{ Anterior disc height $(\mathrm{mm})^{\mathrm{a}}$} \\
\hline Operated level & $12.3 \pm 2.4$ & $12.3 \pm 2.1$ & 0.796 \\
\hline L1-S1 & $56.6 \pm 4.9$ & $56.7 \pm 4.9$ & 0.798 \\
\hline \multicolumn{4}{|c|}{ Posterior disc height $(\mathrm{mm})^{\mathrm{a}}$} \\
\hline Operated level & $7.7 \pm 1.8$ & $7.8 \pm 1.2$ & 0.774 \\
\hline L1-S1 & $36.3 \pm 7.2$ & $36.2 \pm 4.5$ & 0.911 \\
\hline
\end{tabular}

Table 5 Radiographic outcome at midterm follow-up (1 year postoperatively)

\begin{tabular}{llll}
\hline & $\begin{array}{l}\text { Dynesys group } \\
(n=95)\end{array}$ & $\begin{array}{l}\text { PLIF group } \\
(n=81)\end{array}$ & $p$ \\
\hline ROM ( $\left.{ }^{\mathrm{a}}\right)^{\mathrm{a}}$ & $3.5 \pm 1.2$ & $0.9 \pm 0.9$ & $<0.001$ \\
Operated level & $15.3 \pm 4.9$ & $13.1 \pm 3.3$ & 0.001 \\
L1-S1 & $13.1 \pm 2.1$ & $11.9 \pm 2.1$ & $<0.001$ \\
Anterior disc height $(\mathrm{mm})^{\mathrm{a}}$ & $57.1 \pm 7.4$ & $56.6 \pm 4.8$ & 0.670 \\
Operated level & & & \\
1 L1-S1 & $8.8 \pm 1.5$ & $7.8 \pm 1.4$ & $<0.001$ \\
Posterior disc height $(\mathrm{mm})^{\mathrm{a}}$ & $38.0 \pm 7.0$ & $36.7 \pm 5.6$ & 0.171 \\
Operated level & & & \\
L1-S1 & & & \\
\hline Discheight was detern
\end{tabular}

Disc height was determined on radiographs taken with the patient in the neutral position and was assessed by measurement of lines drawn at the most prominent points of the endplates anteriorly or posteriorly. Total disc height measured from L1 to S1 was the sum of the disc height of each level $R O M$ range of motion

${ }^{a}$ Data are displayed as mean \pm SD

posterior disc height of the implanted segment for the PDS group was $7.7 \pm 1.8 \mathrm{~mm}$ preoperatively, $8.8 \pm 1.5 \mathrm{~mm}$ 1 year after surgery, and $8.3 \pm 1.6 \mathrm{~mm}$ in the final followup visit. In the control group, the mean posterior disc height of the implanted segment was $7.8 \pm 1.2 \mathrm{~mm}$ preoperatively, $7.8 \pm 1.4 \mathrm{~mm} 1$ year after surgery, and $7.5 \pm$ $1.3 \mathrm{~mm}$ in the final follow-up visit. The mean posterior disc height of the implanted segment was greater 1 year after Dynesys placement than the preoperative one but then gradually decreased; no statistically significant difference was found between the two groups in the preoperative stage and midterm and final follow-up visit in posterior disc heights of the L1-S1 levels, but there was a statistically significant difference between the two groups in the disc height of the implanted segment 1 year after

Table 6 Radiographic outcome at final follow-up

\begin{tabular}{llll}
\hline & $\begin{array}{l}\text { Dynesys group } \\
(n=95)\end{array}$ & $\begin{array}{l}\text { PLIF group } \\
(n=81)\end{array}$ & $p$ \\
\hline ROM $\left({ }^{\circ}\right)^{a}$ & $3.7 \pm 1.4$ & $0.6 \pm 0.6$ & $<0.001$ \\
Operated level & $15.8 \pm 4.7$ & $13.6 \pm 3.4$ & $<0.001$ \\
L1-S1 & $12.8 \pm 2.2$ & $11.7 \pm 2.2$ & 0.001 \\
Anterior disc height $(\mathrm{mm})^{\mathrm{a}}$ & $57.0 \pm 4.9$ & $56.2 \pm 4.9$ & 0.267 \\
Operated level & & & \\
L1-S1 & $8.3 \pm 1.6$ & $7.5 \pm 1.3$ & $<0.001$ \\
Posterior disc height $(\mathrm{mm})^{\mathrm{a}}$ & $37.9 \pm 6.5$ & $37.0 \pm 4.5$ & 0.281 \\
Operated level & & & \\
\hline L1-S1 & & &
\end{tabular}

Disc height was determined on radiographs taken with the patient in the neutral position and was assessed by measurement of lines drawn at the most prominent points of the endplates anteriorly or posteriorly. Total disc height measured from L1 to S1 was the sum of the disc height of each level $R O M$ range of motion

${ }^{\text {a }}$ Data are displayed as mean \pm SD 
surgery and in the final follow-up visit $(p<0.001)$ (Tables 4 , 5 , and 6).

\section{Overall complication rates}

In the PDS group, perioperative complications occurred in two $(2.1 \%)$ cases, deep venous thrombosis in one case and increasing motor deficit required reoperation in one case. In the PLIF group, perioperative complications occurred in four $(4.9 \%)$ cases. Two deaths occurred intraoperatively, one of which was caused by excessive bleeding and the other one of which was caused by massive myocardial infarction. Two broken screws were found (in two patients) and all of the broken screws have been replaced by reoperation. There was no statistically significant difference between two groups (Table 2).

\section{Discussion}

Fusion is generally considered to be the treatment of choice for painful degenerative conditions of the lumbar spine that have proven unresponsive to nonoperative therapy $[9,10]$. For a long time, good results were thought to be dependent on radiologically confirmed solid fusion. However, several studies in which patients had pseudarthrosis showing the same clinical outcome as patients with solid fusion have challenged this notion [11-13]. Therefore, it is hypothesized that it is the reduction in (rather than the elimination of) segmental motion brought about by partial fusion or perhaps even simply by an alteration of the structure of spinal tissues or by the surgery itself that results in the alleviation of pain. As pain relief represents one of the most important outcomes in achieving a good result after surgery for degenerative spinal disorders, it would thus appear that solid fusion no longer represents a prerequisite for achieving this goal. If this were so, it would present several advantages, including a reduction in the extensiveness of surgery required and the number of complications, as well as the elimination of undesirable late side effects of fusion, such as adjacent segment degeneration [14] and consequent hypermobility [15].

In our study, back and leg pain VAS scores in the PDS group improved from 43.7 to 10.6 and from 51.6 to 6.7, respectively. In the PLIF group, back and leg pain VAS scores decreased from 45.3 to 11.4 and from 52.3 to 7.1, respectively. In the present study, VAS scores for leg and back pain improved in both groups in the final follow-up visit compared with preoperative scores, indicating that both methods were effective for the treatment of degenerative lumbar diseases. As assessed by multiple measures, patients who underwent a PDS performed as well as those who underwent a PLIF. However, this effect is only evident in the result of 3-year follow-up visit. Many studies [16-18] have reported positive results of the Dynesys, while some reports have indicated that results are no better than those obtained with rigid fusion $[19,20]$. We contribute VAS improvement to the biomechanics of the Dynesys, which is a load-sharing device that results in less stiffness than PLIF and may provide immediate stabilization of the diseased segment and neutralize the abnormal forces caused by the pathological bony and soft tissue changes [21-23].

There are conflicting reports on the maintenance of disc height with Dynesys. In our study, changes in posterior disc heights in implant segments showed a contrary pattern of changes compared to those 1 year after surgery and in the final follow-up visit, which were similar to preoperative values. Additionally, CT showed that there was an increase in intervertebral foramen cross-sectional areas and heights immediately after Dynesys implantation but that it subsequently decreased in extent. Thus, no significant difference was evident between preoperative and final follow-up values. It could be concluded from this study that the Dynesys increased spinal canal and intervertebral foramen sizes and local kyphosis with increasing posterior disc height in implanted segments immediately after surgery, but that these returned to their preoperative levels during follow-up visit. However, $\mathrm{Yu}$ found that Dynesys implantation resulted in an increase of posterior disc height and that posterior disc height in the follow-up visit 3 years after surgery was maintained at both the operated level and L1-S1 [21]. Beastall et al. reported a reduction of anterior disc height without a significant increase in posterior disc height 9 months after surgery in 24 patients treated with Dynesys [24]. Kim et al. compared the results of single- and multiple-level stabilization with Dynesys in patients with degenerative spinal disease and found no decrease of disc height in either group with a mean followup visit of $31 \pm 14$ months [25].

In line with the studies of Yu et al. [22], our study demonstrated that both blood loss and the length of hospital stay were significantly less in the Dynesys group. The reduction of blood loss in the Dynesys group is presumably caused by the insertion of the Dynesys device requiring less bone and soft tissue dissection as compared to PLIF. Likewise, the shorter hospital stay of patients in the Dynesys group is most likely due to the insertion of the device which is less invasive as compared to PLIF $[6,26]$. The latter benefits may be of particular importance for elderly patients, or those with significant co-morbidities.

The present study shows the hospital charge in one institution for PDS and PLIF procedures in hospitalization. The results show a significantly higher cost of patients subject to surgical treatment via PLIF fusion than those with PDS. The mean costs of these two procedures differed by US\$1172. The underlying meaning of these results can be interpreted in various ways. First, as the mean hospital cost for both PDS and PLIF surpasses US $\$ 10,000$, a discrepancy of more than US\$1000 in the cost calls into question the real relevance of these data to the economic 
impact of the decision to use one procedure over the other. Our analysis also suggests another nonmonetary means of measuring value. Though hospital charges were significantly greater for PLIF procedure, there may be no significant differences in charges for materials or services in the operating room. This suggests that the greatest difference in charges for these two procedures may arise from perioperative care. Several potential risk factors may influence the perioperative care such as aging and comorbid medical conditions.

Our data showed no significant differences in complications between two groups, although the percentage of complications in the PLIF group (4.9\%) was higher than that in the Dynesys group $(2.1 \%)$. The relatively small sample size may be the reason. Screw loosening and adjacent level instability in the Dynesys group were not significantly greater than those in the PLIF group. Although short-term clinical studies have reported that 6.5 to $20.0 \%$ of patients available for follow-up visit showed screw loosening $[20,27,28]$, screw loosening had no adverse impact on clinical improvement. In our study, only two cases of screw loosening occurred in each group and no revision surgeries were required. The lower rate of screw loosening noted in our study as compared with other previously reported studies might be caused by the fact that patients selected for study were relatively young and had normal bone mineral density. Thus, Dynesys system may be especially effective for younger patients for whom surgical intervention is most needed.

Several potential limitations of this study should be mentioned. First, though the follow-up period in this study was 3 years, a much longer follow-up visit is required to fully understand radiographic and clinical outcomes between PDS and PLIF or determine segmental instability at adjacent levels and long-term outcomes. Moreover, the nature of single-surgeon studies raises the possibility that results may not be externally valid and not reproducible elsewhere. Although there is an advantage, i.e., surgical techniques applied throughout the study were consistent and were not contaminated by multiple surgeons performing surgery with inevitable technique differences, variable implants, and inconsistency in bone grafting techniques with the use of substitutes and osteobiologics. In addition, we also acknowledge limitations caused by our patients' clinical heterogeneity. The difference in age and BMI between two groups may have influence on the result, which will induce bias in the current cohort study.

In conclusion, in this prospective cohort study by a single surgeon, the short-term and long-term functional outcomes clearly demonstrate that PDS have advantages on blood loss, length of stay in hospital, total charge, and radiographic outcomes, but no advantages on leg and back VAS or ODI scores. This indicates that Dynesys stabilization technique requires less extensive surgery than
PLIF but does not result in a superior improvement of the clinical outcome compared with PLIF in the last follow-up visit of a minimum 3-year period.

\section{Competing interests}

The authors declare that they have no competing interests.

\section{Authors' contributions}

The design of the study was done by FJ. HF and JX prepared the manuscript and assisted in the study processes. SW, YX (Xie), and XY (Xu) assisted in the data collections. All authors read and approved the final manuscript.

\section{Acknowledgements}

We thank Dr. Yongyi Xu for his support in obtaining the approval of the ethics committee in this study.

\section{Author details}

'Department of Orthopaedics, Huai'an First People's Hospital, Nanjing Medical University, Huai'an, Jiangsu 223300, People's Republic of China. ²Department of Rehabilitation Medicine, Huai'an Second People's Hospital, Xuzhou Medical College, Huai'an, People's Republic of China.

Received: 5 November 2014 Accepted: 24 May 2015

Published online: 02 June 2015

\section{References}

1. Resnick DK, Choudhri TF, Dailey AT, Groff MW, Khoo L, Matz PG, et al. Guidelines for the performance of fusion procedures for degenerative disease of the lumbar spine. Part 5: correlation between radiographic and functional outcome. J Neurosurg Spine. 2005;2(6):658-61.

2. Kumar MN, Baklanov A, Chopin D. Correlation between sagittal plane changes and adjacent segment degeneration following lumbar spine fusion. Eur Spine J. 2001;10(4):314-9.

3. Okuda S, Iwasaki M, Miyauchi A, Aono H, Morita M, Yamamoto T. Risk factors for adjacent segment degeneration after PLIF. Spine (Phila Pa 1976). 2004;29(14):1535-40.

4. Putzier M, Schneider SV, Funk JF, Tohtz SW, Perka C. The surgical treatment of the lumbar disc prolapse: nucleotomy with additional transpedicular dynamic stabilization versus nucleotomy alone. Spine (Phila Pa 1976). 2005;30(5):E109-14.

5. Lee SE, Park SB, Jahng TA, Chung CK, Kim HJ. Clinical experience of the dynamic stabilization system for the degenerative spine disease. J Korean Neurosci Society. 2008;43(5):221-6.

6. Welch WC, Cheng BC, Awad TE, Davis R, Maxwell JH, Delamarter R, et al. Clinical outcomes of the Dynesys dynamic neutralization system: 1-year preliminary results. Neurosurg Focus. 2007;22(1):E8.

7. Parker MJ, Roberts CP, Hay D. Closed suction drainage for hip and knee arthroplasty. A meta-analysis. J Bone Joint Surg Am. 2004;86-A(6):1146-52.

8. Ovadia D, Luger E, Bickels J, Menachem A, Dekel S. Efficacy of closed wound drainage after total joint arthroplasty. A prospective randomized study. J Arthroplasty. 1997;12(3):317-21.

9. Andrews CL. Evaluation of the postoperative spine: spinal instrumentation and fusion. Semin Musculoskelet Radiol. 2000;4(3):259-79.

10. Nakai S, Yoshizawa H, Kobayashi S. Long-term follow-up study of posterior lumbar interbody fusion. J Spinal Disord. 1999;12(4):293-9.

11. Agazzi S, Reverdin A, May D. Posterior lumbar interbody fusion with cages: an independent review of 71 cases. J Neurosurg. 1999:91(2 Suppl):186-92.

12. Andersen T, Christensen FB, Hansen ES, Bunger C. Pain 5 years after instrumented and non-instrumented posterolateral lumbar spinal fusion. Eur Spine J. 2003;12(4):393-9.

13. France JC, Yaszemski MJ, Lauerman WC, Cain JE, Glover JM, Lawson KJ, et al. A randomized prospective study of posterolateral lumbar fusion. Outcomes with and without pedicle screw instrumentation. Spine (Phila Pa 1976). 1999;24(6):553-60

14. Guigui $P$, Wodecki $P$, Bizot $P$, Lambert $P$, Chaumeil G, Deburge A. Long-term influence of associated arthrodesis on adjacent segments in the treatment of lumbar stenosis: a series of 127 cases with 9-year follow-up. Rev Chir Orthop Reparatrice Appar Mot. 2000;86(6):546-57.

15. Chou WY, Hsu CJ, Chang WN, Wong CY. Adjacent segment degeneration after lumbar spinal posterolateral fusion with instrumentation in elderly patients. Arch Orthop Trauma Surg. 2002;122(1):39-43. 
16. Fay LY, Wu JC, Tsai TY, Wu CL, Huang WC, Cheng H. Dynamic stabilization for degenerative spondylolisthesis: evaluation of radiographic and clinical outcomes. Clin Neurol Neurosurg. 2013;115(5):535-41.

17. Li Z, Li F, Yu S, Ma H, Chen Z, Zhang H, et al. Two-year follow-up results of the Isobar TTL Semi-Rigid Rod System for the treatment of lumbar degenerative disease. J Clin Neurosci. 2013;20(3):394-9.

18. Hoppe S, Schwarzenbach O, Aghayev E, Bonel H, Berlemann U. Long-term outcome after monosegmental $L 4 / 5$ stabilization for degenerative spondylolisthesis with the Dynesys device. J Spinal Disord Tech. 2012.

19. Haddad B, Makki D, Konan S, Park D, Khan W, Okafor B. Dynesys dynamic stabilization: less good outcome than lumbar fusion at 4-year follow-up. Acta Orthop Belg. 2013;79(1):97-103.

20. Grob D, Benini A, Junge A, Mannion AF. Clinical experience with the Dynesys semirigid fixation system for the lumbar spine: surgical and patient-oriented outcome in 50 cases after an average of 2 years. Spine (Phila Pa 1976). 2005;30(3):324-31.

21. Yu SW, Yen CY, Wu CH, Kao FC, Kao YH, Tu YK. Radiographic and clinical results of posterior dynamic stabilization for the treatment of multisegment degenerative disc disease with a minimum follow-up of 3 years. Arch Orthop Trauma Surg. 2012;132(5):583-9.

22. Yu SW, Yang SC, Ma CH, Wu CH, Yen CY, Tu YK. Comparison of Dynesys posterior stabilization and posterior lumbar interbody fusion for spinal stenosis L4L5. Acta Orthop Belg. 2012;78(2):230-9.

23. Fayyazi AH, Ordway NR, Park SA, Fredrickson BE, Yonemura K, Yuan HA. Radiostereometric analysis of postoperative motion after application of dynesys dynamic posterior stabilization system for treatment of degenerative spondylolisthesis. J Spinal Disord Tech. 2010;23(4):236-41.

24. Beastall J, Karadimas E, Siddiqui M, Nicol M, Hughes J, Smith F, et al. The Dynesys lumbar spinal stabilization system: a preliminary report on positional magnetic resonance imaging findings. Spine (Phila Pa 1976). 2007;32(6):685-90

25. Kumar A, Beastall J, Hughes J, Karadimas EJ, Nicol M, Smith F, et al. Disc changes in the bridged and adjacent segments after Dynesys dynamic stabilization system after two years. Spine (Phila Pa 1976). 2008;33(26):2909-14.

26. Schnake KJ, Schaeren S, Jeanneret B. Dynamic stabilization in addition to decompression for lumbar spinal stenosis with degenerative spondylolisthesis. Spine (Phila Pa 1976). 2006;31(4):442-9.

27. Bothmann M, Kast E, Boldt GJ, Oberle J. Dynesys fixation for lumbar spine degeneration. Neurosurg Rev. 2008;31(2):189-96.

28. Stoll TM, Dubois G, Schwarzenbach O. The dynamic neutralization system for the spine: a multi-center study of a novel non-fusion system. Eur Spine J. 2002;11 Suppl 2:S170-8.

\section{Submit your next manuscript to BioMed Central and take full advantage of:}

- Convenient online submission

- Thorough peer review

- No space constraints or color figure charges

- Immediate publication on acceptance

- Inclusion in PubMed, CAS, Scopus and Google Scholar

- Research which is freely available for redistribution 